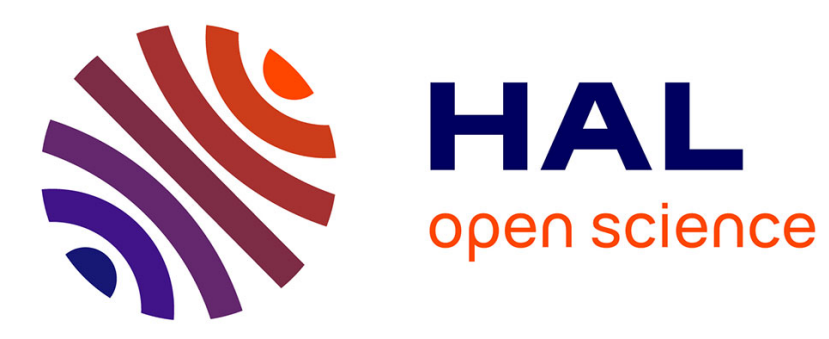

\title{
High-speed confined granular flows down smooth inclines: scaling and wall friction laws
}

\author{
Yajuan Zhu, Renaud Delannay, Alexandre Valance
}

\section{To cite this version:}

Yajuan Zhu, Renaud Delannay, Alexandre Valance. High-speed confined granular flows down smooth inclines: scaling and wall friction laws. Granular Matter, 2020, 22 (4), pp.82. 10.1007/s10035-020-

01053-7 . hal-02960214

\section{HAL Id: hal-02960214 \\ https://hal.science/hal-02960214}

Submitted on 28 Dec 2020

HAL is a multi-disciplinary open access archive for the deposit and dissemination of scientific research documents, whether they are published or not. The documents may come from teaching and research institutions in France or abroad, or from public or private research centers.
L'archive ouverte pluridisciplinaire HAL, est destinée au dépôt et à la diffusion de documents scientifiques de niveau recherche, publiés ou non, émanant des établissements d'enseignement et de recherche français ou étrangers, des laboratoires publics ou privés. 


\title{
High-speed confined granular flows down smooth inclines: Scaling and wall friction laws
}

\author{
Yajuan Zhu • Renaud Delannay - Alexandre Valance
}

the date of receipt and acceptance should be inserted later

\begin{abstract}
Recent numerical work has shown that highspeed confined granular flows down smooth inclines exhibit a rich variety of flow patterns, including dense unidirectional flows, flows with longitudinal vortices and supported flows characterized by a dense core surrounded by a dilute hot granular gas [1]. Here, we further analyzed the results obtained in [1]. More precisely, we characterize carefully the transition between the different flow regimes, including unidirectional, roll and supported flow regimes and propose for each transition an appropriate order parameter. Importantly, we also uncover that the effective friction at the basal and side walls can be described as a unique function of a dimensionless number which is the analog of a Froude number: $F r=V / \sqrt{g H \cos \theta}$ where $V$ is the particle velocity at the walls, $\theta$ is the inclination angle and $H$ the particle holdup (defined as the depth-integrated particle volume fraction). This universal function provides a boundary condition for granular flows running on smooth boundaries. Additionally, we show that there exists a similar universal law relating the local friction to a local Froude number $F r^{l o c}=V^{l o c} / \sqrt{P^{l o c} / \rho}$ (where $V^{l o c}$ and $P^{l o c}$ are the local velocity and pressure at the boundary, respectively, and $\rho$ the particle density) and that the latter holds for unsteady flows.
\end{abstract}

\section{Introduction}

The scientific community has paid particular attention to gravitational granular flows over the past 20 years. These flows are ubiquitous in natural and industrial processes [2,3]. However, their modeling and understanding still leave us with open issues. The complex-

Institut de Physique de Rennes, CNRS UMR 6251, Univ Rennes, 35042 Rennes CEDEX, France ity arises from grain-grain interactions, and also from grain-boundary interactions which may induce correlations over distances much greater than a grain diameter.

The inclined plane geometry was the most employed configuration to study gravity-driven granular flow [4, 5]. It is simple and relevant for many practical situations, but it can be also seen as a rheological test with constant friction. To date, experiments [4] and simulations [6] have focused mainly on mildly sloping and bumpy planes, leading to slow and dense flows which are now fairly well understood [2,7]. More complex flows, including span-wise vortices $[8,9,10]$, were obtained at slightly higher angles suggesting that upon further steepening, granular flows may reveal original features.

Obtaining steady and fully developed (SFD) flows at steep angles is both an experimental and numerical challenge. Indeed, for unconfined flows, there is in general a limit angle above which flows keep accelerating. This limit angle may depend on many parameters such as the mechanical properties of the grain and the nature of the base (flat or bumpy). A simple way to obtain SFD flows at high angles is to introduce frictional side walls. Indeed, if the grainwall friction coefficient is high enough, one may expect that the base friction supplemented by the sidewall friction will be able to balance the driving component of the weight. This is what has been done recently by Brodu et al. [1] by means of discrete element method simulations. These simulations showed that SFD flows can be produced at high angles and revealed the existence of new flow regimes characterized by complex internal structures with heterogeneous particle volume fraction and secondary flows $[1,11,12]$. One of these regimes, referred to as supported flow, is particularly interesting since it displays uncommonly high bulk velocity, 
the granular flow being supported on a dilute granular gaseous layer of highly agitated grains. Similarly to an air-cushion suspension, this layer reduces the effective wall friction and increases significantly the bulk velocity. These "supported" flows are particularly interesting with respect to geophysical issues. The reduction in the effective friction due to the gaseous granular layer could indeed explain unexpected long run-out distances of large granular avalanches.

In this paper, we further analyzed the results obtained by Brodu et al [1] on high-speed confined granular flows. We describe in details the transition between the different flow regimes, including unidirectional, roll and supported flow regimes and provide a unified picture to describe the variation of the effective friction at the boundaries, in terms of a Froude number defined as $F r=V / \sqrt{g H \cos \theta}$ where $V$ is the particle velocity at the walls, $\theta$ is the inclination angle and $H$ the particle holdup (defined as the depth-integrated particle volume fraction). This universal function can be seen as a boundary condition for granular flows running on smooth boundaries. In addition, we find that a similar universal law relating the local friction to a local Froude number $F r^{l o c}=V^{l o c} / \sqrt{P^{l o c} / \rho}$ (where $V^{l o c}$ and $P^{l o c}$ are the local velocity and pressure at the boundary, respectively, and $\rho$ the particle density) can be established and that the latter holds for unsteady flows.

The outline of the paper is the following. In section 2 we briefly present the flow geometry and the discrete element method used for the simulations. Then, in section 3 we recall the different steady and fully developed flow regimes and their main properties. Section 4 is devoted to the detailed analysis of the transition between the different flow regimes. In section 5 , we focus on the basal and sidewall friction and discuss their relationship with velocities at the boundary. Finally, we conclude in section 6 .

\section{Flow geometry}

We consider gravity-driven chute flows with flat frictional bottom and side walls, as shown in Fig.1. The chute is inclined with an angle $\theta$ with respect to the horizontal. $(0 x)$ is the main direction flow, $(0 y)$ the crosswise direction and $(0 z)$ is the direction perpendicular to the flow base. This geometry is similar to that used in $[1,11,13,14]$. Here, the simulation cell has similar dimensions as those employed by Brodu and co-workers $[1,11]$. In particular, the longitudinal length $L$ and the gap $W$ between the side-walls are set to $L=20 D$ and $W=68 D$, respectively (where $D$ is the particle diameter). The channel is not bounded in the $(0 z)$ direction and periodic boundary conditions are employed in the stream-wise direction $(0 x)$.

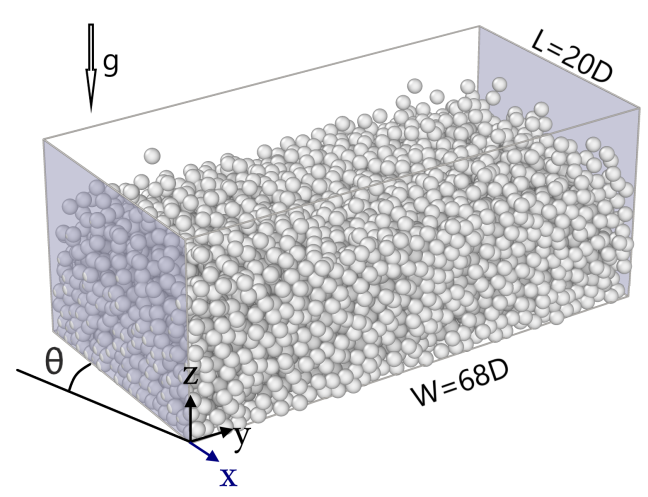

Fig. 1 Scheme of the simulated system. The channel consists of frictional and flat bottom and sides and is inclined with an angle $\theta$ with respect to the horizontal. The longitudinal length $L$ and width $W$ of the channel are set to $20 D$ and to $68 D$, respectively. The channel is not bounded in the $(0 z)$ direction and we use periodic boundary conditions in the stream-wise direction.

We use soft-sphere molecular dynamics simulations where particles in contact can overlap $[1,11]$. The contact forces between two particles have both a normal and a tangential component. The normal force, $F_{n}$, is modeled by a spring and a dashpot: $F_{n}=k_{n} \delta+\gamma_{n} \dot{\delta}$, where $\delta$ is the overlap and $\dot{\delta}$ its derivation with respect to time, respectively, and, $k_{n}$ and $\gamma_{n}$ are the spring stiffness and the viscous damping coefficient, respectively. A similar model is used for the tangential component enforced by the Coulomb friction $\left|F_{t}\right|<\mu\left|F_{n}\right|$ where $\mu$ is the friction coefficient.

We employ the same mechanical parameters as those in the experiments by Louge et al. [15] and in the numerical simulations of Brodu and co-workers [1,11]. We choose values for $k_{n}$ and $\gamma_{n}$ (resp. $k_{t}$ and $\gamma_{t}$ ) such that the normal restitution coefficient $e_{g}^{n}$ (resp. the tangential one $e_{g}^{t}$ ) is equal to $e_{g}^{n}=0.972$ (resp. $e_{g}^{t}=0.25$ ). The particle-particle friction coefficient is set to $\mu_{g}=0.33$.

The walls (i.e., the bottom and the side-walls) are treated like spheres of infinite mass and radius. The normal restitution coefficient $e_{w}^{n}$ and the friction coefficient $\mu_{w}$ for the grain-wall interaction are set to $e_{w}^{n}=0.8$ and $\mu_{w}=0.593$, respectively. These values are also taken from Louge's experiments [15].

The control parameters of the simulation are the mass holdup $H$ and the inclination angle $\theta$, while the the channel width $W$ is kept fixed (i.e., $W=68 D$ ). The particle hold-up $H$, defined as the depth-integrated particle volume fraction (i.e., $H=\int_{0}^{\infty} \phi(z) d z$, where $\phi$ is the particle volume fraction at height $z$ averaged over 
the width and length of the cell) is varied from $4 D$ to $12 \mathrm{D}$, and the inclination from $15^{\circ}$ to $50^{\circ}$.

In the following, unless otherwise specified, particle volume fraction, velocity and velocity fluctuations are averaged spatially in the stream-wise direction and over time during 30 time units (i.e., $\sqrt{D / g}$ ). We mainly focus on steady and fully developed (SFD) flow regimes, that are flows with an averaged velocity that is timeindependent.

\section{Steady and Fully developed flow regimes}

\subsection{General description of the flow regimes}

In [1], 5 different steady and fully developed flow regimes were identified: (i) A unidirectional, dense and layered flow; (ii) a dense and layered flow regime with two longitudinal vortices located at the side wall and close to the free surface; (iii) a roll regime which exhibits a pair of counter-rotative longitudinal vortices that spans the entire width of the cell; (iv) and (v) two types of unusual flows characterized by a dense core floating over a dilute basal layer (referred here after to as supported regimes). Four of these regimes are illustrated in Fig. 2 for $H=6 D$ where the two-dimensional particle volume fraction map in the cross-section of the flow are presented together with the streamlines.

Additional features are worth mentioning. While the unidirectional flow presents a layered structure (see Appendix A for further details) with a high particle volume fraction (i.e., the depth-averaged volume fraction is close to 0.6 ), the roll regime exhibits a slight density inversion, that is a lower particle volume fraction close to the bottom and a higher volume fraction in the bulk flow (see Fig. 2e). The appearance of the longitudinal rolls can be explained as the result of a "RayleighBénard"-like instability [9]. This roll regime has been observed in discrete numerical simulations for the first time for unconfined geometries [10] (i.e., with absence of lateral walls). Our simulations indicate that the lateral confinement does not prevent from the emergence of the roll regime. Interestingly, with the gap width used here (i.e., $W=68 D$ ), we always get a single pair of rolls. We could however conjecture that flow configurations with a much larger gap width should give rise to the formation of several pairs of rolls. In our configuration, the pair of rolls always exhibit the same direction of rotation, leading to a downward motion of the particles in the center of the cell and a upward motion at the lateral walls.

Supported flows exhibit striking feature with a dense core floating on a dilute basal layer. This regime has been first uncovered by Brodu et al. [1]. In comparison (a)

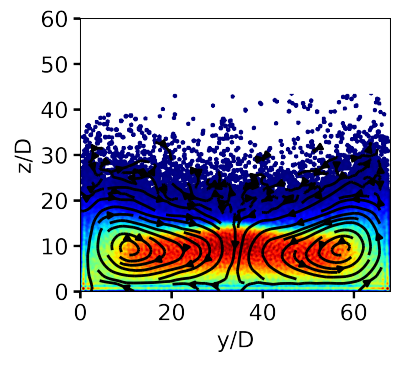

(c)

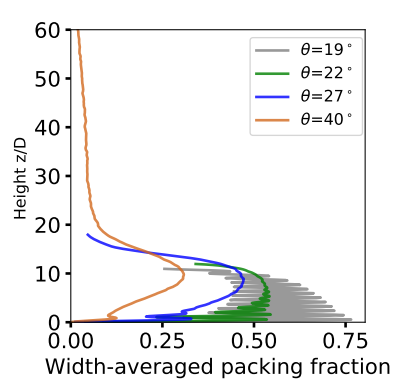

(e)

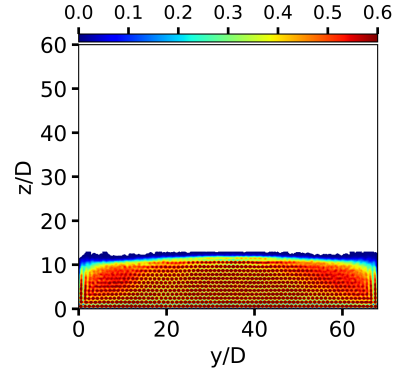

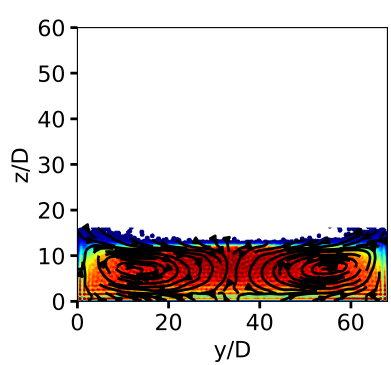

(b)

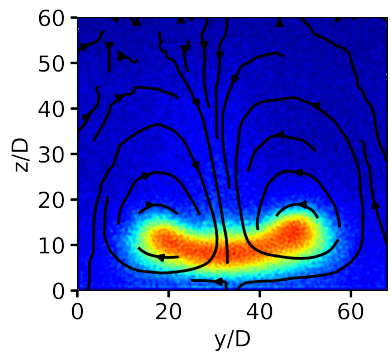

(d)

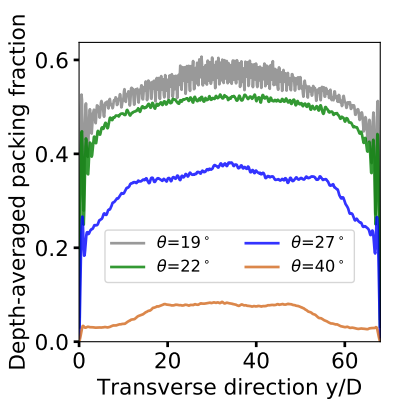

(f)
Fig. 2 Volume fraction map in the cross-section of the flow for different flow regimes with a fixed particle hold-up $(H=6 D)$. The color codes the volume particle fraction (blue indicates dilute regions while red dense ones) and the solid lines stands for the streamlines. (a) Unidirectional, dense and layered flow $\left(\theta=19^{\circ}\right)$; (b) Roll regime $\left(\theta=22^{\circ}\right)$; (c) and (d) supported flow regimes with a symmetric core $\left(\theta=27^{\circ}\right)$ and an asymmetric core $\left(\theta=40^{\circ}\right)$, respectively. (e) and (f) Correspond vertical and transverse profiles of the packing fraction. Note that the density map for the asymmetric core regime has been averaged only over one time unit instead of 30 in order to emphasize the asymmetry and its consequences.

with the roll regime, the density inversion of the volume fraction profile is much more marked (see Fig. 2e) and the core get denser with increasing angle as detailed in the next section. As argued in [1], the appearance of a dense core is possibly linked to the clustering instability observed in the cooling process of granular gas $[16,17]$ and in vibrated granular systems $[18$, 19]. Gravity-driven flows are of course somewhat different from vibro-fluidized granular systems but share some similarities. The former can be seen as the re- 


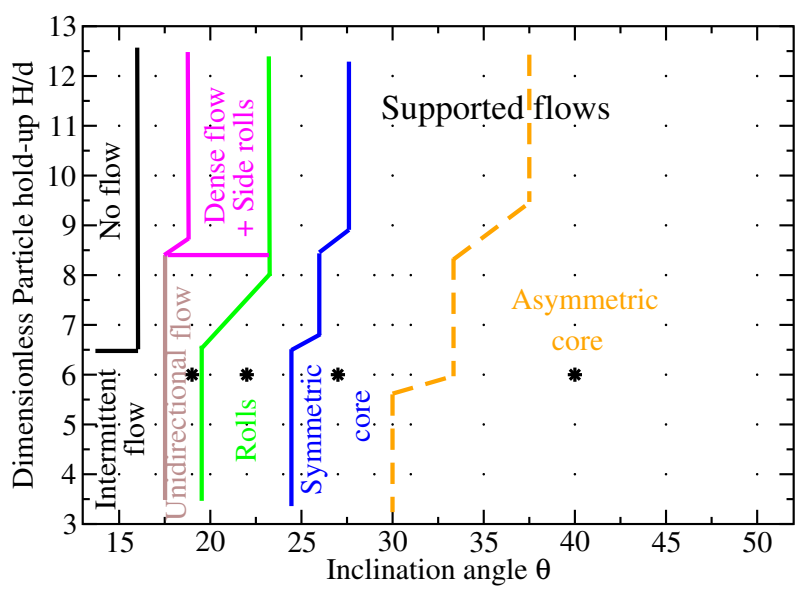

Fig. 3 Phase diagram in the parameter space $(H, \theta)$ for $W=68 D$. Unidirectional and dense flows with layering observed close to the base; Dense flow regime with longitudinal vortices located at the flow surface and close to the side walls; Flows with a pair of longitudinal rolls that spans over the entire cell width; Supported flows with a symmetric dense core; Supported flows with an asymmetric dense core. The black asterisk correspond to the flow regimes illustrated in Fig. 2.

sult of two effects: a shearing, induced by gravity and collisions with the boundaries, which act as an energy source. Thus, the motion along the flow, mainly driven by gravity, could be considered as independent from the motion in the transverse direction, which, according to this hypothesis, would be mainly driven by the interactions of the grains with the boundaries.

Additionally, it is important to note that the longitudinal rolls are still present in the supported flow regime and are not suppressed by the presence of the dense core. They give rise to particle exchange between the dense core and the dilute surrounding region. At the onset of the supported regime (i.e., $\theta=25^{\circ}$ for $H=6 D)$, the core possesses two planes of symmetry, a vertical and an horizontal one. However, for larger inclination angles (i.e., $\theta>30^{\circ}$ ), the horizontal symmetry is broken and the core get bended. As a result, the core starts to rock back and forth. This transition will be discussed in further detail in the next section.

The above flow regimes are all steady and fully developed: they have an averaged velocity $V_{L}$ which is time-independent. Each of them has a limited domain of existence in the parameter space $(H, \theta)$ as illustrated in Fig. 3. Several remarks follow. First, at low angles (i.e., $\theta \leq 17^{\circ}$ ), the flow is not steady: the mean flow velocity does not reach a steady value but fluctuates a lot. These flows are close to the jamming transition and have been named as intermittent flows. Brodu et al. [1] indicated that flows definitively stop below $\theta_{\min } \approx 14^{\circ}$. Second, we can note that the inclination angle is the main parameter which drives the transition of the differ- ent flow regimes. As the inclination angle is increased, several transitions occurs successively: at roughly $20^{\circ}$ unidirectional flows give rise to roll regime which itself leads to supported flow above $25^{\circ}$. The critical angles characterizing these transitions increases slightly with increasing particle hold-up. We will describe carefully these transitions in Section 4.

\subsection{Velocity and granular temperature}

Vertical and transverse profiles of the stream-wise particle velocity for different flow regimes are displayed in Fig. 4. As expected, the flow velocity increases with increasing angle. We can note however that the increase is not only due to an increase of the shear rate but also to a large augmentation of the velocity at the boundaries. It is important to realize that the velocity is discontinuous in the two first layers close to the walls (in particular for moderate inclination angles), indicating that the latter play a peculiar role. This discontinuity may pause a problem to define the relevant slip velocity. However, the velocity difference between the first and second layer remains small and never exceeds a few $\sqrt{g D}$. In the vertical direction, the flow is sheared over the whole flow depth at low inclination angles (i.e., $19^{\circ}$ and $20^{\circ}$ ), while the shear zone is essentially localized in the dilute layer close to the bottom at higher angles (i.e., for supported flows), In the transverse direction, similar features are observed. At low inclination angles, the flow is sheared almost uniformly over the whole width. In contrast, at larger angles, the shear rate is more pronounced in the dilute layer close to the vertical walls than within the dense core. At $\theta=40^{\circ}$, the dense core flows as a plug and does not exhibit any shear within it.

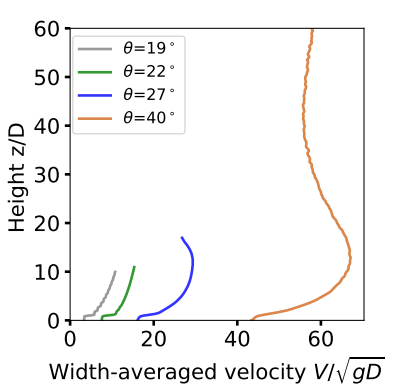

(a)

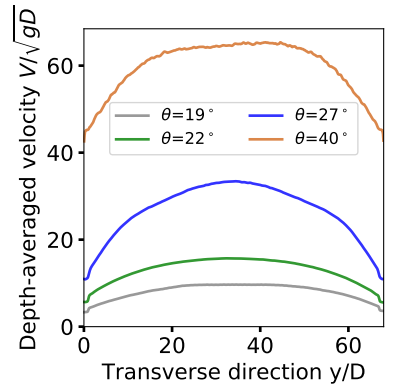

(b)
Fig. 4 (a) Vertical and (b) transverse profiles of the streamwise particle velocity for $\theta=19,20,27$ and $40^{\circ}$ and a fixed particle hold-up $H=6 D$. 


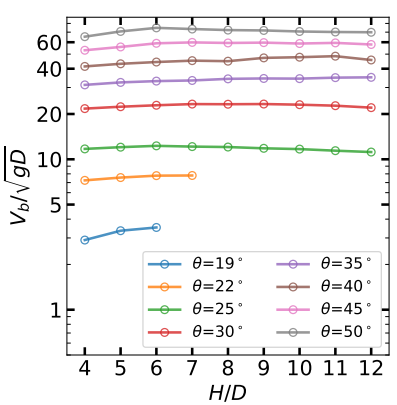

(a)

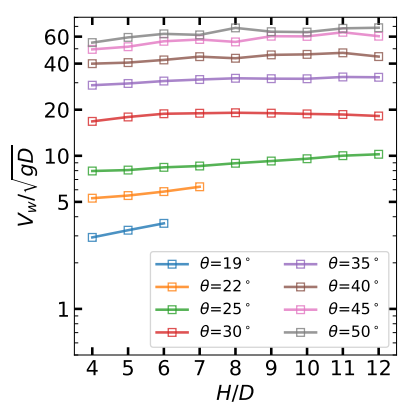

(b)
Fig. 5 (a) Particle velocity $V_{b}$ and $V_{w}$, respectively at the base and at the side-walls, as function of the particle hold-up $H$ for increasing inclination. Both velocities are almost invariant with the particle hold-up $H$, except for small inclination angles where a slight increase is observed. In contrast, they increases significantly with increasing inclination angle.

Importantly, we confirm the scaling law proposed by Brodu et al. [1] concerning the mean flow velocity $V_{L}$ :

$\frac{V_{L}}{\sqrt{g D}} \approx A(H / D)^{\alpha}\left(\sin \theta-\sin \theta_{c}\right)$

with $\alpha \approx 0.25, A \approx 122$ and $\theta_{c} \approx 17.5^{\circ}$. One can note that the value of the critical angle $\theta_{c}$ is very close to the lower boundary for SFD flows (see Fig. 3). The values of the fitting parameters $A$ and $\theta_{c}$ are expected to be dependent both of the channel width $W$ and the microscopic friction coefficients $\mu_{g}$ and $\mu_{w}$. This scaling indicates that the mean velocity increases both with the inclination angle and the particle hold-up. However, it is important to note that the increase of the mean flow velocity with the particle hold-up is rather mild and drastically differs from the Bagnold scaling law (i.e., $V_{L} \propto H^{3 / 2}$ ) which is relevant for slow and dense granular flows on bumpy bottoms. For the latter, the particle velocity almost vanishes at the bumpy bottom while our flows that run over a smooth base have a finite and large velocity at the base. We believe that the difference in the scaling law results essentially from the different nature of the basal boundary condition.

In addition to the mean flow velocity, the velocities at the boundaries are also interesting and relevant quantities. The basal and side-wall slip velocities are calculated at $z=0.5 D$ and at $y=0.5 D$, respectively, that is within the first wall particle layer. Note that this choice differs from that made in [11], where the basal slip velocity was evaluated in the second particle layer at $z=1.5 \mathrm{D}$. Although velocities are discontinuous in the two first layers, their difference remains small and both definitions of the slip velocity leads to similar results. The velocity at the bottom and at the side walls are found to be almost independent of the particle hold-up within the range investigated so far (i.e., $4<H / D<12)$ and increases with increasing inclination angle (see Fig. 5). Interestingly, they are quantitatively similar and are linearly correlated with the rescaled flow velocity $V_{L} /(H / D)^{0.25}$ :

$$
V_{b} \approx V_{w} \approx V_{L} /(H / D)^{0.25} \approx A\left(\sin \theta-\sin \theta_{c}\right) \sqrt{g D} .
$$

This invariance with the particle hold-up is quite surprising but it is in line with the fact that the mean velocity $V_{L}$ moderately increases with $H$. It is important to note that this invariance is well verified for supported flows but does not hold for flows with small inclination angles (i.e., $\theta<25^{\circ}$ ), including both unidirectional and dense flows and the roll regime. For the latter, we observe a clear increase of $V_{b}$ and $V_{w}$ for increasing particle hold-up.

Granular temperature is a measure of the particle velocity fluctuations. It is an important parameter in various theories aiming to capture granular flow behaviors. It is defined as $T=\left(T_{x x}+T_{y y}+T_{z z}\right) / 3$ where $T_{i j}=<u_{i} u_{j}>-<u_{i}><u_{j}>, u_{i}$ is the $i$ component of the instantaneous particle velocity and $<$. $>$ stands for time averaging and spatial averaging in the streamwise direction. We provide in Fig. 6 temperature map within the cross-section of the flow as well as vertical and transverse profiles of the temperature for various flow regimes. We observe contrasting features for slow and large angles, respectively. For unidirectional flows, the temperature is relatively homogeneous with a temperature at the bottom slightly greater than within the bulk flow (see Fig. 6.a). In the roll regime, the temperature is still very homogeneous within the bulk flow but there is a larger contrast of temperature between the bottom temperature and the bulk one. For large angles (i.e., for supported flows), the temperature map exhibits contrasting features. The supported dense core is very cold and surrounded by a dilute hot gas. This flow regime thus displays strong heterogeneities of temperature which is strongly correlated to particle volume fraction.

Lastly, it should be noted that the granular temperature profile exhibits discontinuities close to the boundaries, similarly as the velocity profile: the first wall layer is generally much colder than the second layer, emphasizing that the two first wall layers play an important role. 


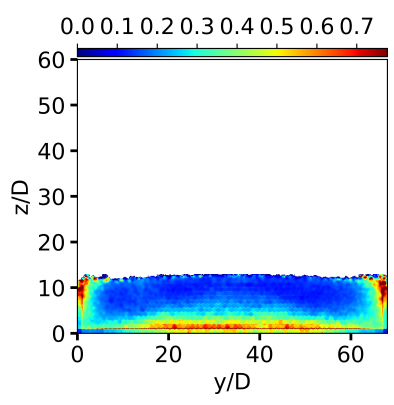

(a)

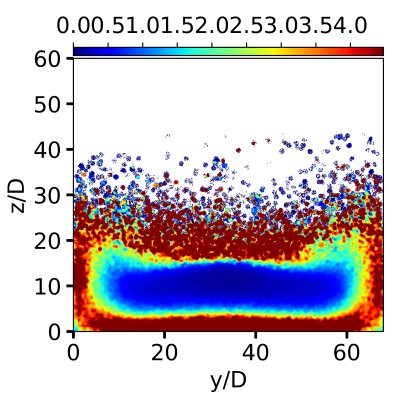

(c)

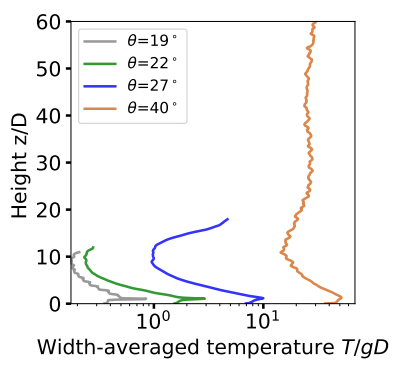

(e)

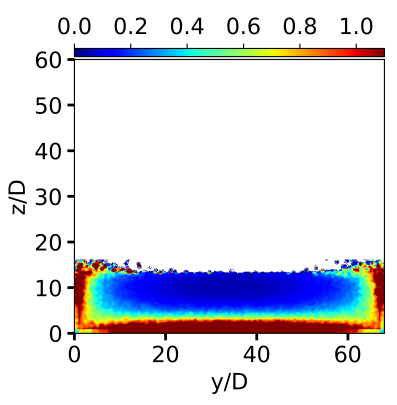

(b)

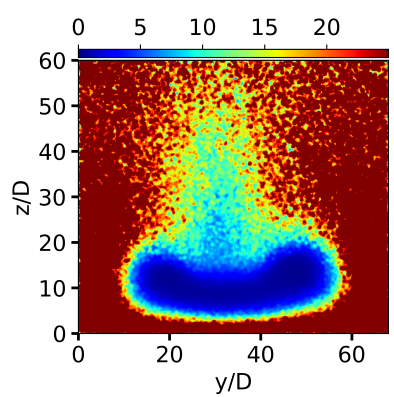

(d)

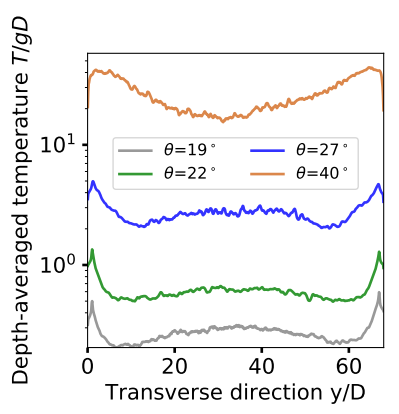

(f)
Fig. 6 Temperature map for different flow regimes. $H=$ $6 D$ and $W=68 D$. (a) Unidirectional dense regime; $(\theta=$ $\left.19^{\circ}\right)$; (b) Roll regime $\left(\theta=22^{\circ}\right)$ : (c) and (d) supported flows: symmetric core $\left(\theta=27^{\circ}\right)$ and asymmetric core $\left(\theta=40^{\circ}\right)$; (e) Corresponding vertical and (f) transverse profiles of the granular temperature.

\section{Flow regime transition}

In this section, we describe the transition between the different flow regimes. For that purpose, we investigate the variation of several key parameters that highlight the flow regime transition.

\subsection{Vorticity}

We first consider the transition from the unidirectional flow regime towards the roll regime. The vorticity is the natural quantity for characterizing the presence of longitudinal vortices. It is defined as $\boldsymbol{\Omega}=\nabla \times \mathbf{v}$.

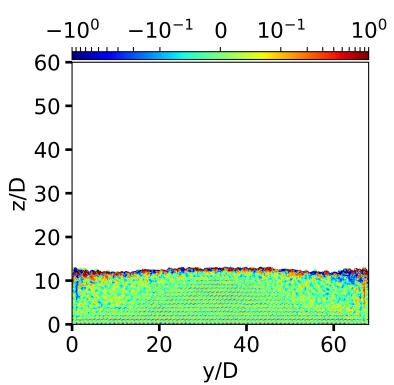

(a)

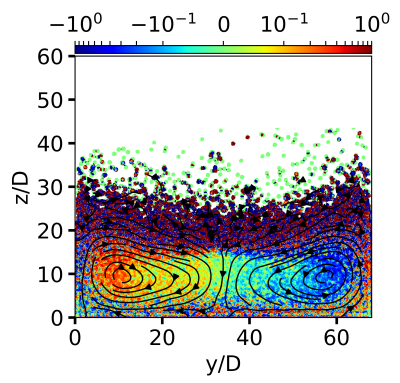

(c)

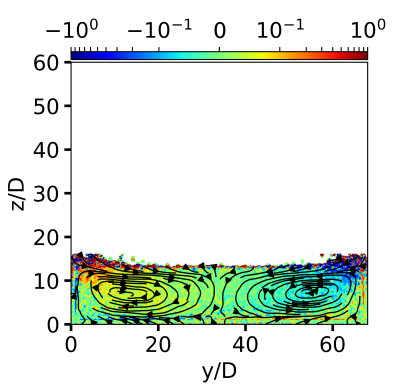

(b)

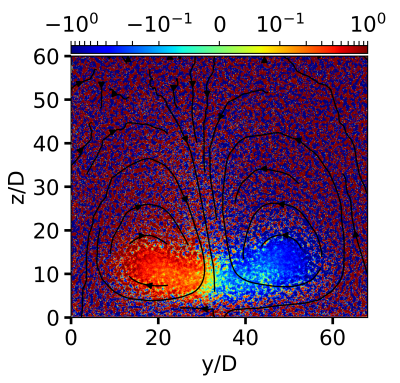

(d)
Fig. 7 Vorticity map for different flow regimes. $H=6 D$ and $W=68 D$. Solid lines represents the streamline in the flow cross-section. (a) Unidirectional dense regime; $\left(\theta=19^{\circ}\right)$; (b) Roll regime $\left(\theta=22^{\circ}\right)$ : (c) and (d) supported flows: symmetric core $\left(\theta=27^{\circ}\right)$ and asymmetric core $\left(\theta=40^{\circ}\right)$.

In Fig. 7, we present the vorticity map for different flow regimes. For unidirectional flows, (e.g., $H=6 D$ and $\theta=19^{\circ}$ ), the vorticity is close to zero (i.e., less than $\left.2.10^{-2} \sqrt{g / D}\right)$. Upon increasing inclination angle (i.e., for $\theta=22^{\circ}$ ), roll regime develops with a visible pair of counter-rotative longitudinal vortices (see Fig. 7.b). Upon further increase of the inclination angle (i.e., for $\theta=27^{\circ}$ and $40^{\circ}$ ), the flow exhibits similar vorticity pattern but with increasing values of the vorticity. The behavior is illustrated in Fig. 8 that displays the maximum value of the vorticity within the vortex as a function of the inclination angle for various particle hold-up. This plot confirms that for dense undirectional flows $\left(\theta<20^{\circ}\right)$ the maximum vorticity is extremely small and start to increase at the onset of the roll regime. Besides, one can note that the vorticity increases with the inclination angle at a greater rate when one enters the supported regime (i.e., for $\theta \approx 25^{\circ}$ ) and tends to saturate at large angles corresponding to the asymmetric core regime (i.e., $\theta>30^{\circ}$ ). 


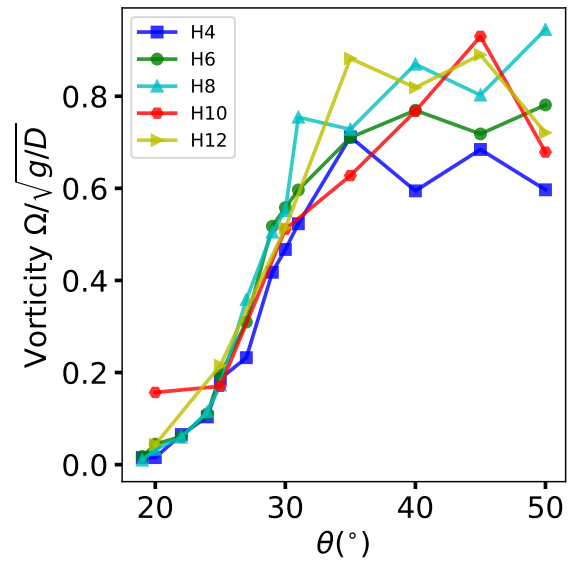

Fig. 8 Vorticity versus inclination angle for various particle hold-up. The value of the vorticity stands for the maximum value of the vorticity within the rolls (see Fig. 7).

As a summary, we confirmed that the vorticity is the appropriate parameter to delineate the transition from the dense flow regime towards the roll regime.

\subsection{Maximum packing fraction}

We then focus on the transition from the roll regime towards the supported regime. As the supported regime is accompanied with the formation of a dense core, it is then natural to investigate how the volume fraction evolves with increasing inclination angle.

In Fig. 9, we present the maximum value of the volume fraction $\phi_{\max }$ in the cross-section of the flow as a function of the inclination angle. For a given particle hold-up, this value first decreases with increasing angle, as naturally expected. However, we observe a critical angle around $25^{\circ}$ at which the decrease is stopped and the packing fraction reaches a local minimum. Above this critical angle, the maximum packing fraction increases with increasing angle and eventually reaches a peak value at $\theta \approx 30^{\circ}$ before decreasing again. The appearance of the local minimum coincides with the emergence of the supported flow regime with a dense core floating on a gaseous layer. As previously discussed, the increase of the packing fraction can be seen as the signature of the clustering instability in granular gas [16].

Importantly, the local maximum of the packing fraction is reached just before the transition towards the asymmetric core regime. After the local maximum, the packing fraction starts a new decrease with increasing angle. This decrease is concomitant with a shrinkage of the latter: particles from the core evaporate and enter the surrounding gaseous region.

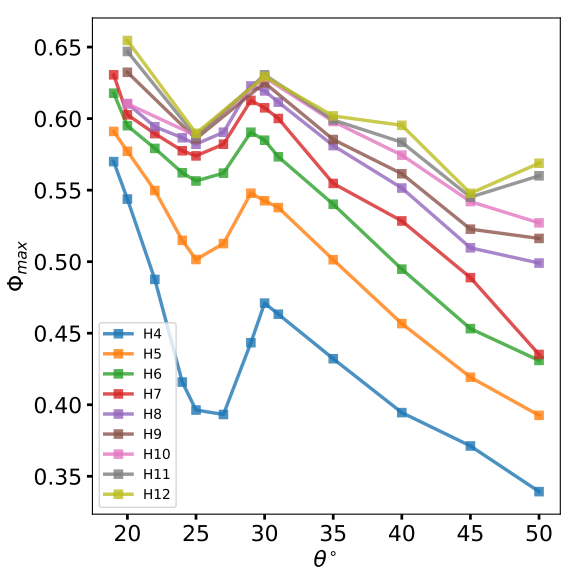

Fig. 9 Maximum packing fraction $\phi_{\max }$ as a function the inclination angle for various particle hold-up.

Upon increasing the particle hold-up, the same trend is observed for the maximum packing fraction. The packing fractions at the local minima and maxima both increase with increasing particle hold-up but the difference between the maximum and minimum packing fraction tends to decrease. This behavior of the packing fraction is reminiscent of the liquid-gas first-order transition of a molecular gas. There is indeed a striking resemblance with the isothermal curves of a simple gas in the pressure-volume diagram.

As a conclusion, the evolution of the maximum packing fraction $\phi_{\max }$ as a function of the inclination provides a clear indicator of the transition between the roll regime and the supported flows. The minimum of the curve $\phi_{\max }(\theta)$ delineates the onset of the supported flow regimes.

\subsection{Skewness}

The last transition concerns the supported regime with a asymmetric core. We attempted to characterize the asymmetry of the dense core by investigating the asymmetry of the instantaneous depth-integrated transverse packing fraction profiles $\phi(y)$ through the skewness parameter $S$ defined as

$S=\frac{\int_{0}^{W} d y \phi(y)(y-\mu)^{3} /\left(\int_{0}^{W} d y \phi(y)\right)}{\left[\int_{0}^{W} d y \phi(y)(y-\mu)^{2} /\left(\int_{0}^{W} d y \phi(y)\right)\right]^{3 / 2}}$

with $\mu=\int_{0}^{W} d y \phi(y) y / \int_{0}^{W} d y \phi(y)$.

We present in Fig. 10.a the skewness as a function of time. The skewness $S$ is small at low and moderate inclination angles (e.g., $\theta=19,22$ and $27^{\circ}$ ) but becomes significant at larger inclination angle (e.g., $\theta \geq 40^{\circ}$ ) and oscillates between positive and negative values with a 


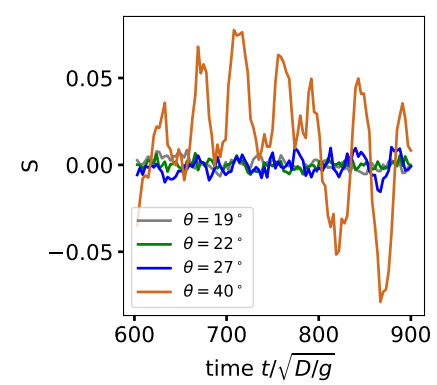

(a)

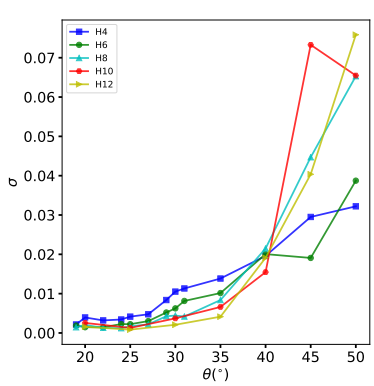

(b)
Fig. 10 (a) Evolution of the skewness $S$ of the depthintegrated transverse packing fraction profile $\phi(y)$ as function of time for inclination angles $\theta=19,22,27$ and $40^{\circ}$ and a fixed particle hold-up $H=6 D$. (b) Standard deviation of the skewness $S$ as a function of the inclination angle for various particle hold-up.

well defined periodicity which is directly related to the rocking motion of the dense core. The variation of the amplitude of the skewness as a function of the inclination is shown in Fig. 10.b for various particle holdup $H$. The amplitude is negligible (i.e., smaller than $0.005)$ for low inclinations and starts to increase progressively at the onset of the appearance of the supported regime (i.e., for $\theta>25^{\circ}$ ). This means that the asymmetry of the flow develops as soon as the supported regime emerges. However, the amplitude of the asymmetry reaches a significant value for larger inclination angles. For definiteness, we have set the onset of the transition when the amplitude of the asymmetry goes beyond the critical value $\sigma_{c}=0.01$. With this criterion, we find that the asymmetric transition slightly increases with increasing particle hold-up: it occurs at $\theta \approx 30^{\circ}, 35^{\circ}$ and $37^{\circ}$ for $H=4 D, 8 D$ and $12 D$, respectively. This parameter thus allows to delineate a transition between supported regimes with a symmetric and asymmetric core, respectively.

\section{Sidewall and basal friction}

In these types of confined flows, boundaries play an important role. It is thus instructive to investigate in particular how the effective sidewall and bottom friction, defined as the ratio of tangential to normal stresses, evolve according to the flow regimes reported below. Brodu and co-workers [1] showed the sidewall and bottom friction both increase with increasing inclination angle but surprisingly decrease with increasing particle hold-up. Here, we are going further by investigating how these trends could be cast into simple laws.

We first investigate the averaged effective friction at the basal and lateral walls computed from the ratio of the wall-averaged tangential stress to the wall-averaged normal stress. Second, we analyze the basal and lateral friction at the local scale.

\subsection{Averaged friction law}

The salient outcome of our data analysis is that the variation of both the averaged basal and sidewall friction can be simply described through a unique dimensionless number, analog to a Froude number, $\mathrm{Fr}=$ $V_{\text {boundary }} / \sqrt{g H \cos \theta}$, where $H$ is the particle hold-up, $\theta$ the angle of inclination and $V_{\text {boundary }}$ the velocity at the considered boundary (i.e., either $V_{b}$ or $V_{w}$ ). Indeed, if we plot the effective basal friction and sidewall friction as a function of the Froude number $\mathrm{Fr}$ for all the SFD flow regimes investigated so far (i.e., within the parameter range: $4 \leq H / D \leq 12$ and $15^{\circ} \leq \theta \leq 50^{\circ}$ ), we get a nice collapse of all the data onto a unique curve (see Fig. 11). The $\mu(F r)$ curve increases mono-

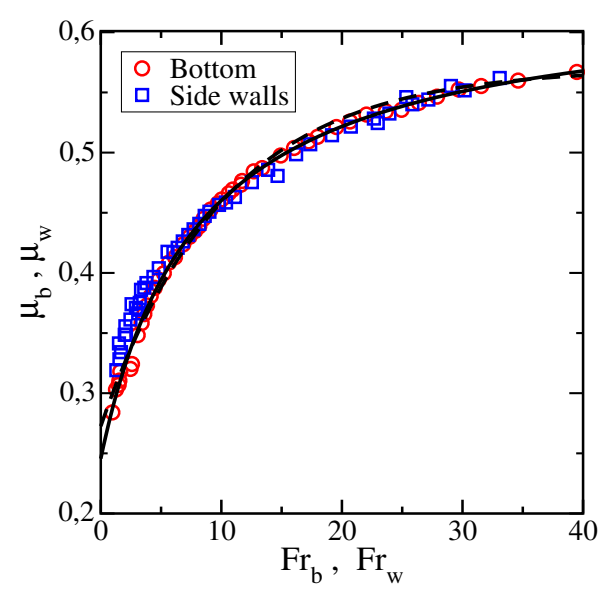

Fig. 11 Effective basal friction $\mu_{b}$ (circle symbols) and sidewall friction $\mu_{w}$ (square symbols) as a function of the Froude number $F r_{b}=V_{b} / \sqrt{g H \cos \theta}$ and $F r_{w}=V_{w} / \sqrt{g H \cos \theta}$, respectively, for all the SFD flow regimes investigated so far (i.e., within the parameter range: $4 \leq H / D \leq 12$ and $15^{\circ} \leq$ $\theta \leq 50^{\circ}$ ). All the data collapse on a unique master curve (solid line) which is obtained by a fit using Eq. 4 . An exponential fit (dash line) of the form $\mu(F r)=\mu_{2}+\left(\mu_{1}-\mu_{2}\right) \exp \left(-F r / F r_{0}\right)$ with $\mu_{1} \approx 0.27, \mu_{2}=0.57$ and $F r_{0} \approx 10.1$ works well too.

tonically with the Froude number and seems to saturate at large Froude number to an asymptotic value. Interestingly, the $\mu(F r)$ curve shares strong resemblance with the $\mu(I)$ rheological curve for dense granular flows over bumpy bottoms and can be well approximated by a similar functional form:

$\mu(F r)=\mu_{1}+\frac{\mu_{2}-\mu_{1}}{1+F r_{0} / F r}$, 
where $\mu_{1}, \mu_{2}$ and $F r_{0}$ are fitting parameters. The best fit to the data provides $\mu_{1} \approx 0.24, \mu_{2} \approx 0.63$ and $F r_{0} \approx$ 8. Formally, the parameters $\mu_{1}$ and $\mu_{2}$ correspond to the value of the wall friction for vanishing and large Froude number, respectively. For the basal friction law, $\theta_{1}=\arctan \left(\mu_{1}\right) \approx 13.5$ has the same physical meaning as the corresponding parameter in the $\mu(I)$ rheology and stands for the minimum inclination angle at which a flow is sustainable. This value is smaller but reasonably close to the critical inclination angle inclination below which the flow stops: $\theta_{\text {min }} \approx 14^{\circ}$ [11]. Similarly, $\theta_{2}=\arctan \left(\mu_{2}\right)$ can be interpreted as the maximum inclination angle at which steady flows can be achieved in absence of side-wall friction. Physically, this value is bounded by the microscopic friction angle between the particle and the wall (i.e., $\theta_{w}=\arctan \left(\mu_{w}\right)=30.7^{\circ}$ ). The best fit gives $\theta_{2} \approx 32.2^{\circ}$ which is greater than the theoretical upper bound $\theta_{w}$. This means that the functional form we employ, although it provides a good approximation in the range of studied Froude number, is probably not fully relevant. A functional form based on the exponential law,

$\mu(F r)=\mu_{2}+\left(\mu_{1}-\mu_{2}\right) \exp \left(-F r / F r_{0}\right)$,

seems to provide a better alternative. This functional form is similar to the original friction law proposed by Pouliquen for flows over bumpy bases in [4]. However, it differs in a subtle aspect. The original Pouliquen's friction law phrased as a function of the Froude number would be written as $\mu=\mu_{1}+\left(\mu_{2}-\mu_{1}\right) \exp \left(-F r_{0} / F r\right)$. This form has the same asymptotic values for vanishing and large Froude number $\left(\mu_{1}\right.$ and $\mu_{2}$, respectively) but has a different slope at zero Froude number: the slope is zero whereas with the form given by Eq. 5 the slope is finite. A detailed analysis at vanishing Froude number would be required to determine which is the better form. With the functional form given by Eq. 5, the best fit gives $\mu_{1} \approx 0.27, \mu_{2}=0.57$ and $F r_{0} \approx 10.1$. This fit provides a value of $\mu_{2}$ which is satisfactorily smaller than the upper bound $\mu_{w}=0.593$.

Several additional comments follow. (i) It is important to note that the $\mu_{b}\left(F r_{b}\right)$ law $\left(\operatorname{resp} . \mu_{w}\left(F r_{w}\right)\right)$ should be considered as a boundary condition which relates the effective friction at smooth boundaries to the flow velocity at the boundaries (through the Froude number $F r_{b}$ or $F r_{w}$ ). Consequently, they do not have the same status as the frictional $\mu(I)$ rheology which relates the internal effective friction (between adjacent granular layers parallel to the base) to the local internal shear rate through the inertial number $I$.

(ii) The Froude numbers $F r_{b}$ and $F r_{w}$ are defined from the flow velocity evaluated at the boundary (i.e., $V_{b}$ or $\left.V_{w}\right)$ because we are interested in what occurs at the boundaries. Our definition of the Froude number thus differs from the one used in related works $[4,20,21]$ and which is based on the mean flow or free surface velocity. If we use the latter definition for the Froude number, we also obtain a good collapse but with a slightly larger scattering. This result is not surprising since we have seen that the mean flow velocity is of the same order of magnitude as the velocities at the boundaries (see Eq. 2). In contrast, dense and slow flows over a bumpy bottom have a vanishing basal velocity (i.e., noslip condition) and thus the latter does not scale with the mean flow velocity.

(iii) It is worthwhile to highlight the similarities and differences between the $\mu_{b}\left(F r_{b}\right)$ law and the $\mu(I)$ rheological law obtained for dense flows over a bumpy bottom. As already mentioned, the latter describes the internal effective friction as a function of the local inertial number $I$ or equivalently to the local shear rate. By continuity of the stresses, one can deduce a relationship between the friction and the inertial number at the base, respectively $\mu_{b}$ and $I_{b}$. The $\mu(I)$ rheology thus provides a relationship between the basal friction and $I_{b}$ while the $\mu_{b}\left(F r_{b}\right)$ law relates the basal friction to the flow velocity at the base through the Froude number $F r_{b}$. The question that arises is how the basal inertial number $I_{b}$ is related to the Froude number $F r_{b}$. For dense flows over bumpy bottom, the basal velocity is generally assumed to vanish such that the Froude number $F r_{b}$ is reduced to zero. This contrasts with flows on smooth inclines, where the flow velocity $V_{b}$ at the base is finite and closely related to the mean flow velocity $V_{L}$ $\left(V_{b} \propto V_{L} / H^{1 / 4}\right)$. The relation between $F r_{b}$ and $I_{b}$ in the context of granular flows on smooth inclines is not known in general, except for unidirectional and dense flows. In the latter regime, the bulk flow can be still reasonably well described by the $\mu(I)$ rheology as shown in [11] (this is not the case for the other flow regimes), while the basal friction was shown to obey the $\mu_{b}\left(F r_{b}\right)$ law. The continuity of the friction at the bottom provides us with the following relation $\mu\left(I_{b}\right)=\mu_{b}\left(F r_{b}\right)$, which thus relates $I_{b}$ and $F r_{b}$.

(iv) The $\mu(F r)$ friction law provides a simple explanation for the decrease of the bottom and wall friction with increasing particle hold-up. Indeed, recalling that the velocities at the boundaries are almost invariant with the particle hold-up (see Eq. 2), the Froude number decreases with increasing particle hold-up at a fixed inclination angle. This results in a decrease of the basal friction since $\mu(F r)$ is an increasing function of the Froude number.

(v) Eq. 4 or Eq. 5 together with Eq. 2 and the definition of the Froude number provides us with an explicit 
expression of the basal and sidewall friction as a function of the inclination angle and particle hold-up.

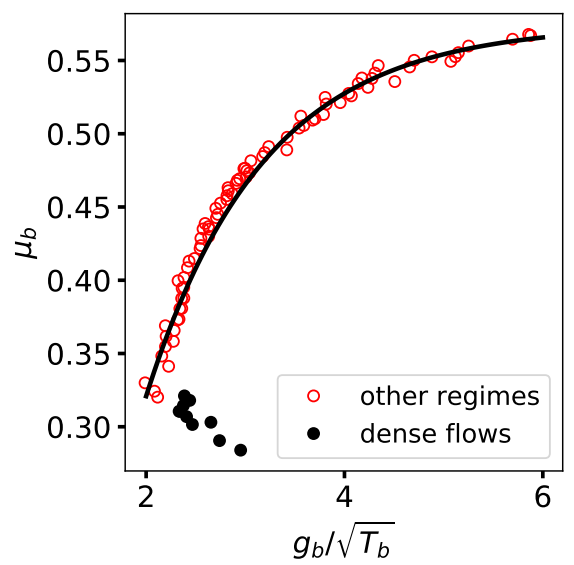

Fig. 12 Bottom friction $\mu_{b}$ as a function of the dimensionless contact slip velocity $g_{b} / \sqrt{T_{b}}$. The solid line represent the best exponential fit of the form: $\mu_{b}=\mu_{2}+\left(\mu_{1}-\right.$ $\left.\mu_{2}\right) \exp \left[-0.85\left(g_{b} / \sqrt{T_{b}}-1.86\right)\right]$ with $\mu_{1} \approx 0.32$ and $\mu_{2}=0.575$. The data corresponding to unidirectional dense flows do not fall on the master curve represented by the solid line.

(vi) In kinetic theories for granular flows, the effective friction at bumpy wall is often expressed as a function of the dimensionless quantity $V / \sqrt{T}$ [22]. In the case of flat frictional wall [22], the relevant quantity is $g / \sqrt{T}$ where $g=\|\mathbf{V}-(D / 2) \boldsymbol{\omega} \times \mathbf{n}\|$ is the contact slip velocity at the wall ( $\mathbf{n}$ is the unit vector normal to the wall and $\boldsymbol{\omega}$ is the mean angular velocity). It is thus instructive to check whether the friction at the basal and side walls can be also described in terms of the ratio $g / \sqrt{T}$. We present in Fig. 12 the effective bottom friction as a function of the dimensionless quantity $g_{b} / \sqrt{T_{b}}$ calculated at the base. We find a nice collapse of the data on a single curve which is very similar to the $\mu(F r)$ curve. We can note however a deviation of the monotonic behavior at low value of the friction (i.e., at small inclination angles corresponding to dense flows). It thus turns out that the Froude number and the dimensionless contact slip velocity $g / \sqrt{T}$ play a similar role and are closely related. We find indeed the following correlation:

$F r_{b} \approx 9.95\left(\frac{g_{b}}{\sqrt{T_{b}}}-1.86\right)$.

The above correlation works well for large Froude number but fails for small Froude number below 2, corresponding to dense flows.

(vii) Interestingly, we noted that if we use a definition of the Froude number based on the contact slip velocity $g_{b}$ (resp. $\left.g_{w}\right)$ instead of the translational velocity $V_{b}$ (resp. $\left.V_{w}\right)$, we also obtain a nice collapse of

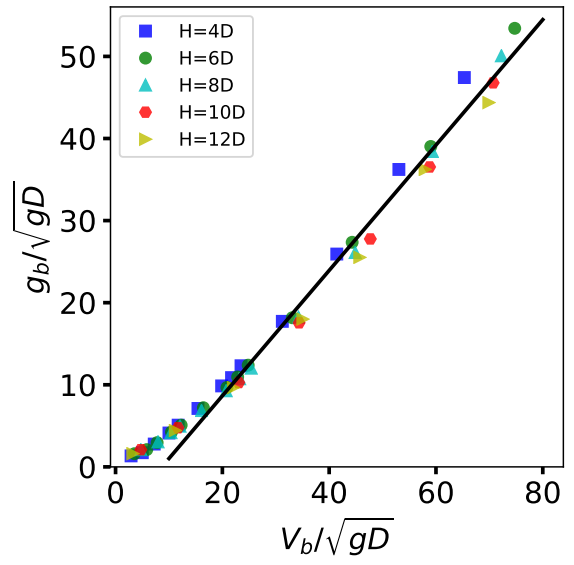

Fig. 13 Relationship between the contact slip velocity $g_{b}$ and the translational velocity $V_{b}$ at the base for increasing particle hold-up from $H=4 D$ to $H=12 D$. The solid line is an affine fit to the data.

the data on a unique master curve. This confirms that there is a close relationship between the contact slip velocity and translational velocity at the boundaries (see Fig. 13).

(vii) Finally, the exact form of the $\mu(F r)$ curve is expected to be dependent of the material properties of the grains and the walls and in particular of the microscopic friction coefficients $\mu_{w}$ (resp. $\mu_{g}$ ) between the particles and the walls (resp. between the particles). Preliminary numerical investigations indicate that the scaling with the Froude number is however preserved when changing the material properties of the grains and the walls. In other words, only the fitting parameters $\mu_{1}, \mu_{2}$ and $F r_{0}$ are sensitive to a change of the micro-mechanical parameters.

\subsection{Local friction law}

In the previous subsection, we analyzed the effective friction at the base and at the side-walls at a global scale. It can be interesting to check whether the friction law established previously also holds at a local scale. The effective friction at the base and at the side-walls are not uniform along the cross-wise direction (y) and the depth (z), respectively, as illustrated in Fig. 14. The basal wall friction is smaller close to the side-walls than in the center of the channel. The influence of the sidewall extends over $10 D$ to $20 D$. Similarly, side-wall friction exhibits a strong gradient according to the depth: it decreases with increasing depth and reaches its minimum value at the base.

Defining a local Froude number (i.e., $F r^{l o c}=V^{l o c} / \sqrt{P^{l o c} / \rho}$, where $V^{l o c}$ and $P^{l o c}$ are the local particle velocity and pressure, respectively, at a given location at the basal or 


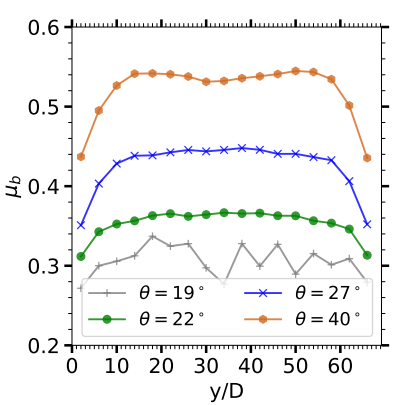

(a)

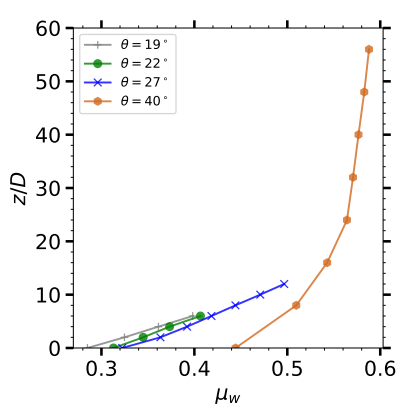

(b)
Fig. 14 (a) Cross-wise profiles of the effective basal friction $\mu_{b}(y)$ for $H=6 D$ and various inclination angles. (b) Vertical profiles of the effective sidewall friction $\mu_{w}(z)$ for $H=6 D$ and various inclination angles.

side wall, and $\rho$ is the particle density), we can display how the local friction varies as a function of the local Froude number. Similarly, as for the global friction, we obtain for both the local basal and side-wall friction a nice collapse on a unique master curve $\mu^{l o c}\left(\mathrm{Fr}^{l o c}\right)$ (see Fig. 15.a). This curve differs slightly from the global law $\mu(F r)$ essentially at large Froude number. Using an exponential fit (cf Eq. 5), we obtained slightly different values for the fitting parameters: $\mu_{1}^{\text {loc }} \approx 0.25$ $\mu_{2}^{l o c} \approx 0.59$ and $\mathrm{Fr}_{0}^{l o c} \approx 9.6$. The most significant difference concerns the value of $\mu_{2}$ (0.59 against 0.57$)$ which is closer to the upper bound $\mu_{w}=0.593$.

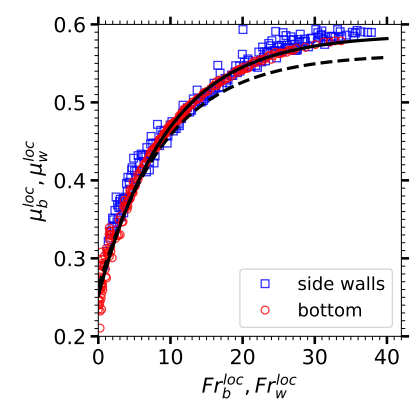

(a)

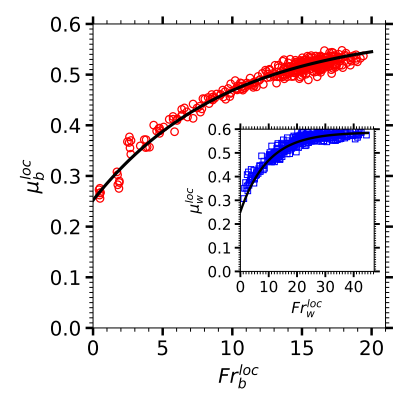

(b)
Fig. 15 (a) Local basal friction $\mu_{b}^{l o c}$ and sidewall friction $\mu_{w}^{l o c}$ as a function the local Froude number $F r_{b}^{l o c}=V_{b}^{l o c} / \sqrt{P_{b}^{l o c} / \rho}$ and $F r_{w}^{l o c}=V_{w}^{l o c} / \sqrt{P_{w}^{l o c} / \rho}$, respectively. The solid line stands for the best exponential fit while the dash line represents the exponential fit for the global friction $\mu(F r)$. (b) Evolution of the local basal friction in the transient regime for $H=$ $10 D$ and $\theta=40^{\circ}$ as a function of the local Froude number $F r_{b}^{l o c}=V_{b}^{l o c} / \sqrt{P_{b}^{l o c} / \rho}$. The solid line stands for the master local friction curve established in (a). Inset: Evolution of the local side-wall friction in the transient regime for $H=10 D$ and $\theta=40^{\circ}$ as a function the local Froude number $F r_{w}^{l o c}=$ $V_{w}^{l o c} / \sqrt{P_{w}^{l o c} / \rho}$.
Interestingly, the local friction law seems to hold also for unsteady flows. As detailed in [1], the flow exhibits a transient before reaching a steady state: the mean velocity increases monotonically in course of time and eventually saturates to a steady value. Figure 15.b shows that even during the transient regime, the local friction both at the base and at the side-walls evolves along the master local friction curve $\mu^{l o c}\left(\mathrm{Fr}^{l o c}\right)$. This result thus indicates that the local friction laws are robust features for both steady and unsteady flows.

\section{Conclusion}

We have studied high-speed confined granular flows down smooth inclines and describe in detail the different SFD flow regimes, including unidirectional dense flows, roll regime and supported flows. We have identified key parameters that allows to delineate precisely the domain of existence of the different flow regimes in the parameter space $(H, \theta)$. Importantly, we have highlighted that the friction at the basal and side walls can be described by a unique curve that depends solely of the Froude number defined as $V / \sqrt{g H \cos \theta}$, where $V$ is the particle velocity at the walls. We showed additionally that the friction at the local scale also obeys a unique law as a function of the local Froude number defined as $F r^{l o c}=V^{l o c} / \sqrt{P^{l o c} / \rho}$. This local friction law is shown to be very robust since it holds both for steady and unsteady flows. We thus strongly believe that the local friction law may be used successfully as a reliable boundary condition for flows running on smooth walls.

A crucial question is the extent to which the SFD flow regimes and their features are specific to the material parameters and the confined geometry that we have considered. Further extensive simulations where the material parameters (friction and restitution coefficient) and channel width $W$ are varied would be required to check the generality of our outcomes. This is a work planned for the near future. Preliminary results show that the SFD flows identified in this paper are very robust to parameter change but their onset of appearance may be significantly affected. For example, increasing the dissipation in the grain-grain collision favors and reinforces the development of supported flows.

Finally, these results provide a unique set of very complex granular flow regimes for testing theoretical and rheological models.

Acknowledgements We acknowledge the support of the French Research National Agency through the project ANR-16-CE010005 . 


\section{A Layering index}

In the undirectional and dense flow regime, the particle volume fraction exhibits strong oscillations. It could be interesting to introduce a layering index $I_{L}$ to quantify the number of oscillation cycles. It is defined as follows. As the wavelength of the oscillation is of the order of one grain diameter, we look at within each layer of one diameter thickness and parallel to the bottom whether the volume fraction oscillates. In

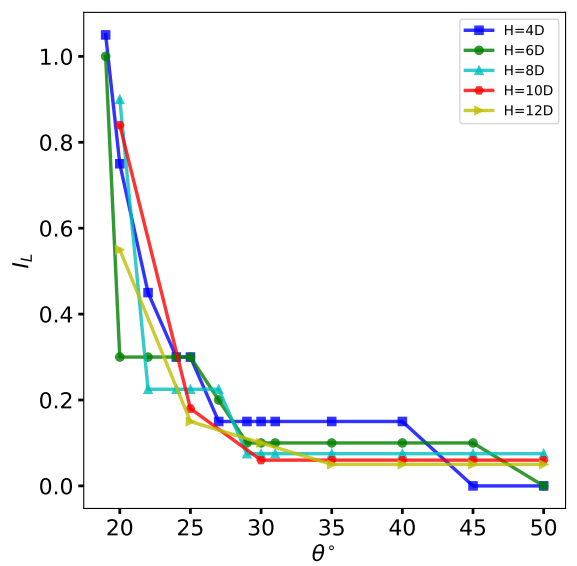

Fig. 16 Layering index $I_{L}$ as a function of the inclination angles for various particle hold-up: $I_{L}=$ $\left(1 / N_{\max }\right) \sum_{i} Y\left(\phi_{i}^{\max }-\phi_{i}^{\min }-0.1\right)$ where $Y$ is the Heaviside function.

each layer $i$, we thus calculate the maximum and the minimum of the volume fraction, $\phi_{i}^{\text {min }}$ and $\phi_{i}^{\max }$, respectively. If the amplitude of the oscillation (i.e., $\phi_{i}^{\max }-\phi_{i}^{\min }$ ) in a given layer is greater than a critical value $\Delta \phi$, the layer is associated to an ordered layer of particles and the layering index is incremented by one unit. The layer index $I_{L}$ is defined as $I_{L}=\left(1 / N_{\max }\right) \sum_{i} Y\left(\phi_{i}^{\max }-\phi_{i}^{\min }-\Delta \phi\right)$ where $Y$ is the Heaviside function and $N_{\max }=H / 0.6$ is the highest possible number of ordered layers within a uniform and dense flow with a mean volume fraction of 0.6 and particle hold-up $H$. The renormalization of $I_{L}$ by $N_{\max }$ provides an index which is bounded by 1 . The critical value $\Delta \phi$ used to quantify the layering is taken to be 0.1 . This choice is somewhat arbitrary but it is good comprise to capture the oscillation of the packing fraction and eliminate random fluctuations of the packing fraction profile.

The variation of the layering index with the inclination angle is shown in Fig. 16. SFD unidirectional and dense flows exhibit a strong layering with a layering index close to 1 , indicating that the whole depth of the flow dense flows) is layered. Upon increasing the inclination angle (from $20^{\circ}$ to $25^{\circ}$ ), the layering index decreases progressively towards zero. Above $25^{\circ}$ (i.e., in the supported flow regime), the layering index has fallen to a small but finite residual value (below 0.2 ). This mean that even in the supported regime, there remains one ordered layer which is located at the bottom. This residual dense ordered layer disappears at very large inclination angle. The layering index can thus not be employed to delineate the transition towards the supported flow regime.

\section{References}

1. N. Brodu, , R. Delannay, A. Valance, P. Richard. New patterns in high-speed granular flows. Journal of Fluid Mechanics 769, 218-228 (2015).

2. G. MiDi. On dense granular flows. European Physical Journal E 14(4), 341 (2004).

3. R. Delannay, A. Valance, A. Mangeney, O. Roche, P. Richard. Granular and particle-laden flows: from laboratory experiments to field observations. Journal of Physics D: Applied Physics 50(5), 053001 (2017).

4. O. Pouliquen. Scaling laws in granular flows down rough inclined planes. Physics of Fluids 11(3), 542 (1999).

5. Y. Forterre, O. Pouliquen. Flows of Dense Granular Media. Annual Review of Fluid Mechanics 40(1), 1 (2008).

6. L.E. Silbert, D. Ertas, G.S. Grest, T.C. Halsey, D. Levine, S.J. Plimpton. Granular Flow down an Inclined Plane Bagnold Scaling and Rheology. Physical review. E, 64, 051302 (2001).

7. R. Delannay, M. Louge, P. Richard, N. Taberlet, A. Valance. Towards a theoretical picture of dense granular flows down inclines. Nature Materials 6, 99 (2007).

8. Y. Forterre, O. Pouliquen. Longitudinal Vortices in Granular Flows. Phys. Rev. Lett. 86, 5886 (2001).

9. Y. Forteree, O. Pouliquen. Stability analysis of rapid granular chute flows: formation of longitudinal vortices. Journal of Fluid Mechanics 467, 361387 (2002).

10. T. Börzsönyi, R.E. Ecke, J.N. McElwaine. Patterns in Flowing Sand: Understanding the Physics of Granular Flow. Phys. Rev. Lett. 103, 178302 (2009).

11. N. Brodu, P. Richard, R. Delannay. Shallow Granular Flows down Flat Frictional Channels: Steady Flows and Longitudinal Vortices. Phys. Rev. E 87, 022202 (2013).

12. J.L. Ralaiarisoa, A. Valance, N. Brodu, R. Delannay. High speed confined granular flows down inclined: numerical simulations. EPJ Web Conf. 140, 03081 (2017).

13. N. Taberlet, P. Richard, A. Valance, W. Losert, J.M. Pasini, J.T. Jenkins, R. Delannay. Superstable Granular Heap in a Thin Channel. Phys. Rev. Lett. 91, 264301 (2003).

14. P. Richard, A. Valance, J.F. Métayer, P. Sanchez, J. Crassous, M. Louge, R. Delannay. Rheology of Confined Granular Flows: Scale Invariance, Glass Transition, and Friction Weakening. Physical Review Letters 101(24), 248002 (2008).

15. M.Y. Louge, S.C. Keast. On dense granular flows down flat frictional inclines. Physics of Fluids 13(5), 1213 (2001).

16. I. Goldhirsch, G. Zanetti. Clustering instability in dissipative gases. Phys. Rev. Lett. 70, 1619 (1993).

17. S. McNamara, W.R. Young. Inelastic collapse in two dimensions. Phys. Rev. E 50, R28 (1994).

18. E. Opsomer, F. Ludewig, N. Vandewalle. Dynamical clustering in driven granular gas. EPL 99(4), 40001 (2012).

19. P. Eshuis, K. van der Weel, M. Alam, M. van der Hoef, H. Kuipers, S. Luding, D. van der Meer, D. Lhose. Buoyancy driven convection in vertically shaken granular matter: experiment, numerics, and theory. Granular Matter 15, 893 (2013).

20. A.J. Holyoake, J.N. McElwaine. High-speed granular chute flows. Journal of Fluid Mechanics 710 (2012).

21. J.M.N.T. Gray, A.N. Edwards. A depth-averaged $\mu(I)$ rheology for shallow granular free-surface flows. Journal of Fluid Mechanics 755, 503534 (2014).

22. J.T. Jenkins. Boundary Conditions for Rapid Granular Flow: Flat, Frictional Walls. Journal of Applied Mechanics 59(1), 120 (1992). 